\title{
Analysis of axial Length of Marfan Syndrome with Bilateral Ectopia Lentis by Z-scores for Chinese Patients under 20 years Old
}

\section{Zexu Chen}

Eye \& ENT Hospital of Fudan University

Jiahui Chen

Eye \& ENT Hospital of Fudan University

Min Zhang

Eye \& ENT Hospital of Fudan University

Jialei Zheng

Eye \& ENT Hospital of Fudan University

Michael Deng

Eye \& ENT Hospital of Fudan University

Tianhui Chen

Eye \& ENT Hospital of Fudan University

Yinghong Ji

Eye \& ENT Hospital of Fudan University

Yongxiang Jiang ( $\nabla$ yongxiang_jiang@163.com )

Eye \& ENT Hospital of Fudan University

\section{Research Article}

Keywords: axial length, Z-score, Marfan syndrome, ectopia lentis

Posted Date: December 7th, 2020

DOl: https://doi.org/10.21203/rs.3.rs-117990/v1

License: @ (1) This work is licensed under a Creative Commons Attribution 4.0 International License. Read Full License 


\section{Abstract}

Purpose: To analyze the axial length (AL) in young patients with Marfan syndrome (MFS) and bilateral ectopia lentis (EL) before the lens surgery.

Methods: This study reviewed MFS patients under 20 with bilateral EL from January 2015 to October 2020. The Z-scores were introduced in terms of the number of standard deviations (SD) from the mean of age-matched normative data. Using Z-scores, the distribution of $A L$ and influence factors were evaluated. The correlations between AL and other biometrics were analyzed.

Results: We reviewed 183 patients, and enrolled both eyes. The mean age was $8.44 \pm 4.69$ years old. The mean AL increased from $23.41 \pm 1.73 \mathrm{~mm}$ under 5 years old to $26.40 \pm 2.28 \mathrm{~mm}$ in the 16-20 age group, and when plotted, the trend presented a logarithmic curvature. The mean Z-AL score was $1.74 \pm 2.68$. One-third of eyes had Z-score $<0$. The right eye and the eye complicated with megalocornea had larger ZAL scores. A positive correlation was found between Z-AL and Z-Corneal Curvature Radius $(r=0.265, P<$ $0.001)$.

Conclusion: Young patents with bilateral EL but small AL should not be excluded from MFS without systematic examination. The age-adjusted Z-score will facilitate further study of the individual variations in $\mathrm{AL}$ across different ages.

\section{Introduction}

As an important component of the ocular refractive media, a normal crystalline lens is critical to the development of the visual system. Ectopia lentis (EL) refers to the dislocation of the lens from its physiological position, which may cause refractive errors of varying severity, poor visual acuity, and amblyopia in young patients ${ }^{1}$. The major etiology of congenital EL is Marfan syndrome (MFS, OMIM 154700), the prevalence of which ranges from 4.6 to 6.5 per $100000^{2-4}$. MFS is an autosomal dominant hereditary disease caused by fibrillin 1 (FBN1) gene mutations ${ }^{5}$; fibrillin 1 is the main constituent of the microfilaments, which are the key structural components of the ciliary zonules ${ }^{6}$. Mutations of $F B N 1$ result in the impaired suspension of the crystalline lens in its anatomic position ${ }^{7}$. In addition to $E L$, the eyes of patients with MFS are characterized by long axial lengths (AL), low corneal curvatures, severe astigmatism, and dense myopia ${ }^{8}$.

Refractive and biometric characteristics of patients with MFS have been reported ${ }^{9-13}$; however, most studies have used a relatively small sample size without control groups. To our knowledge, few investigations have examined the distribution of $A L$ and the correlations between $A L$ and other biometrics before lens surgery in young MFS patients with bilateral EL. In this retrospective study of a relatively large group of patients, we aimed to investigate the overall status of axial development in MFS patients younger than 20 years old and the relationships among AL development and patient demographics, including age, sex, family history, and comorbidities. We introduced the age-adjusted Z-scores by using 
external controls to analyze the correlations of AL development with the corneal curvature radius (CCR), $\mathrm{AL} / \mathrm{CRC}$ ratio, and white-to-white horizontal corneal diameter (WTW). Our results provide a useful reference for the precise planning of surgical interventions for MFS patients with bilateral EL.

\section{Results}

\section{Patient demographics}

The ocular biometric parameters of 183 MFS patients under 20 years old with bilateral EL were collated. Both eyes were enrolled. All participants were Chinese, and $55.49 \%$ were male. The age distribution of the participants is shown in Fig. 1, and the mean age was $8.44 \pm 4.69$ years (range, 2-20 years). Patients under 5 years old constituted the highest proportion: $36.07 \%$ of the total participants. The number of patients decreased with the advancing age group. Subjects were further stratified by age considering the characteristic of AL growth in normal children: < 5 years old, 6-8 years old, 9-11 years old, 12-15 years old, and 16-20 years old.

\section{The AL distribution}

The AL increased as the patients aged. The mean AL was $23.41 \pm 1.73 \mathrm{~mm}$ under 5 years old, $24.30 \pm$ $2.61 \mathrm{~mm}$ in the $6-8$ years of age group, $25.45 \pm 2.67 \mathrm{~mm}$ in the $9-11$ years of age group, $25.62 \pm$ $2.80 \mathrm{~mm}$ in the 12-15 years of age group, and $26.40 \pm 2.28 \mathrm{~mm}$ in the 16-20 years of age group (Fig. 2a). There was a significant difference in the AL among all the age subgroups $(P<0.001)$, and the trend in $A L$ increase shown in the scatterplot follows a logarithmic curve $\left(R^{2}=0.145, P<0.001\right)$ (Fig. $\left.2 b\right)$. Considerable variation in AL was observed among patients of the same age.

\section{The Z-AL score distribution}

The overall mean Z-score was $1.74 \pm 2.68$ (range, -2.55 to 10.68). For the age groups, the mean Z-scores were $1.68 \pm 2.37$ for under 5 years old, $2.02 \pm 3.37$ for the $6-8$ years of age group, $1.91 \pm 2.67$ for the 9 11 years of age group, $1.10 \pm 2.20$ for the $12-15$ years of age group, and $1.74 \pm 2.11$ for the $16-20$ years of age group (Fig. 3a). The age difference was successfully adjusted by the Z-AL score because there were no statistical differences in the Z-AL score among the different age groups $(P=0.531)$ (Fig. 3a). The distribution of AL within the same age group was well-represented by the Z-score. The proportion of the ZAL score shared a similar pattern among the age subgroups. A considerable variation in AL was observed. In all the age groups, about $30 \%$ of patients had a Z-AL score $<0$, which means that some MFS patients had a shorter AL than the age-matched control. Extremely long AL (Z-AL score $\geq 3$ ) was observed in about $30 \%$ of patients (Fig. 3b).

\section{Relationship between Z-AL score with different factors}


To further explore the factors influencing the AL of the participants, we analyzed the relationships among AL or Z-AL score and laterality, sex, and family history (Table 1). The right eyes had slightly but significantly longer $\mathrm{A} L$ than the left ones $(P=0.013)$. Males seemed to have longer $\mathrm{AL}$, but the difference was not significant $(P=0.267)$. There was no difference between patients with or without a family history of MFS ( $P=0.907)$. In the 20 eyes $(10.87 \%)$ with microspherophakia, the AL appeared to be larger, but the difference was not statistically significant $(P=0.145)$. Of the 145 eyes $(39.84 \%)$ with qualified WTW measurements, megalocornea (WTW $\geq 13$ ) was found in $10(6.90 \%)$. MFS eyes complicated by megalocornea had longer $\mathrm{AL}$ on average $(P=0.005)$.

Table 1

Comparison of the AL and Z-AL between different factors according to age groups.

\begin{tabular}{|c|c|c|c|}
\hline \multirow[t]{2}{*}{ Factor } & \multicolumn{3}{|l|}{ Z-AL score } \\
\hline & Number of eyes & Mean \pm SD & $P$-value \\
\hline \multicolumn{4}{|l|}{ Laterality } \\
\hline Right & 182 & $1.85 \pm 2.72$ & \multirow[t]{2}{*}{0.013} \\
\hline Left & 182 & $1.62 \pm 2.64$ & \\
\hline \multicolumn{4}{|l|}{ Sex } \\
\hline Male & 202 & $1.86 \pm 2.76$ & \multirow[t]{2}{*}{0.267} \\
\hline Female & 162 & $1.59 \pm 2.58$ & \\
\hline \multicolumn{4}{|c|}{ Family history (MFS) } \\
\hline Positive & 144 & $1.70 \pm 2.56$ & \multirow[t]{2}{*}{0.907} \\
\hline Negative & 220 & $1.77 \pm 2.76$ & \\
\hline \multicolumn{4}{|c|}{ Microspherophakia } \\
\hline Positive & 20 & $2.53 \pm 2.64$ & \multirow[t]{2}{*}{0.145} \\
\hline Negative & 346 & $1.69 \pm 2.68$ & \\
\hline \multicolumn{4}{|c|}{ Megalocornea } \\
\hline Positive & 10 & $4.72 \pm 3.51$ & \multirow[t]{2}{*}{0.005} \\
\hline Negative & 135 & $1.65 \pm 2.61$ & \\
\hline \multicolumn{4}{|c|}{$\begin{array}{l}P \text {-values }<0.05 \text { are highlighted in bold (laterality, paired Wilcoxon test.). (gender, family history, and } \\
\text { comorbidities, Wilcoxon test). }\end{array}$} \\
\hline
\end{tabular}

\section{Correlation between AL with biometry parameters as Z- scores}


Z-AL scores were plotted against Z-CCR, Z-WTW, AL/CCR ratio, and corneal astigmatism (Fig. 4). There was a positive correlation between Z-AL and Z-CCR $(r=0.265, P<0.001)$ (Fig. 4a) and a positive correlation between Z-AL and AL/CCR ratio $(r=0.762, P<0.001)$ (Fig. 4b). No correlation was found between Z-AL and Z-WTW ( $r=0.052, P=0.537)$ (Fig. 4c). A mild but significant correlation was observed between Z-AL and AST ( $r=0.151, P=0.004)$ (Fig. $4 \mathrm{~d})$.

\section{Discussion}

About $60 \%$ of patients of MFS had EL, which is usually bilateral, progressive, and has a superior direction 8 . The implantation of intraocular lens (IOL) plays a crucial part in the postoperative visual rehabilitation of children. The $A L$ is the most important factor affecting the accuracy of IOL power, and a 1-mm error of measurement can induce an approximate 2.5D refractive error ${ }^{19}$. Previous investigations have shown the $\mathrm{AL}$ in the eyes of patients with EL was different from those in normal eyes ${ }^{20}$. However, there have been few studies of the characteristics of the AL in MFS eyes before lens surgery. In this study, we recruited 183 MFS patients with bilateral EL to investigate the distribution and influence factors of AL in the developing age. The age-adjusted Z-score is a powerful tool to study the influencing factors and the correlation between $\mathrm{AL}$ and other biometrics.

Here, we present the distribution of AL in MFS patients with bilateral EL. Similar to the results for the normative cohort ${ }^{21}$, there was a positive correlation between $\mathrm{AL}$ and age in the current study. $\mathrm{A}$ logarithmic correlation between $\mathrm{AL}$ and age was also previously found in congenital cataract patients ${ }^{22}$. $\mathrm{AL}$ increases dramatically in the first 2 years of life, then grows at a slower rate until adulthood ${ }^{22}$. The AL growth trend for patients in the current study also presented a logarithmic curvature. However, because of the limited number of patients under 3 years old, the high growth rate normally seen in the early stage of the logarithmic curve is absent in this study. Previous studies found that EL can manifest at an early age: EL is recognized at age 0 in $38 \%$ of patients with neonatal MFS ${ }^{23}$, and $12.5 \%$ of MFS patients show EL before 3 years of age ${ }^{8}$. The prevalence might have been underestimated because of the difficulties for young patients expressing themselves and poor cooperation under ophthalmic examination. The distribution of AL among patients under 3 years old is uncertain and warrants further study.

The Z-score has been used by cardiologists to adjust for body surface area when measuring aortic sinus diameters in various individuals ${ }^{24}$, and new Z-scores have been developed to normalize age, sex, height, and weight ${ }^{25}$. A Z-score $\geq 2.0$ ( $\geq 3.0$ for patients under 20 years old) is a major criterium in the diagnosis of MFS ${ }^{14}$. However, the age-adjusted Z-score introduced in the current study was not intended to assist in making a diagnosis based on ocular biometrics, which has been excluded in the 2010 Ghent 2 criteria due to uncertain specificity ${ }^{14}$. In this study, the Z-scores successfully normalized the age differences in AL and other parameters by comparing age-matched published normative values. The distribution of AL across the age groups was well-represented by the Z-score. 
The mean AL was 1.74 standard deviations from the normative mean measurement. About $30 \%$ of eyes had relatively long AL (Z-AL score $\geq 3$ ), and the largest had a Z-score of 10.68. A long AL has been proven to be a risk factor for retinal detachment ${ }^{26}$ and open-angle glaucoma ${ }^{27}$ in the eyes of MFS patients. Thus, patients with longer AL should have more intensive ophthalmic exams, especially when they have a visual complaint. Although a long AL has been recognized as an ocular manifestation of MFS, our study showed that about $30 \%$ of the eyes had a short $\mathrm{AL}$ ( $Z-A L$ score $<0)$, and the proportion was similar among the different age groups. Thus, young patients with bilateral EL but small AL should not be simply diagnosed as having idiopathic EL without considering the systematic features. Since the manifestations of MFS can be atypical at the young age ${ }^{28}$, a genetic test or careful follow-up is strongly recommended.

Considerable variation was observed among those of the same age, indicating that the development of the AL in MFS patients is associated with multiple factors in addition to age. However, the sex and family history of MFS were not associated with differences in AL. The right eye AL was, on average, slightly longer than the left one, with uncertain clinical implications. One possible explanation is that a variety of FBN1 mutations could contribute to this difference. More than 3000 mutations of FBN1 are known of today ${ }^{29}$, but only $14.20 \%$ of the mutations are recurrent ${ }^{30}$. The misfolded fibrillin- 1 and the disorganized elastin in the scleral stroma could lead to stretching of the sclera through the two physiological functions of fibrillin-1 (structure component or TGF- $\beta$ signaling mediator) and by different underlying genetic mechanisms (haploinsufficiency or dominant-negative effect) ${ }^{30}$. In addition, the heterogeneity of FBN1 mutations may lead to various degrees of EL, which accelerates globe growth via defocus or deprivation. Thus, the measurement of $A L$ should be individualized to achieve more precise results, even in patients of the same age. Further genotypic-phenotypic analyses are required to understand the variations in $A L$ and severity of EL. The Z-score will be helpful when analyzing FBN1 mutations and ocular phenotypes in young patients of different ages.

A previous study described the ocular biometrics of MFS eyes based on age stratification ${ }^{31}$. However, although $\mathrm{AL}$ is an important indicator of eyeball development, the relationships between $\mathrm{AL}$ and other biometric parameters have been scarcely reported in MFS patients. Correlations among AL and other biometrics were analyzed in the form of the age-adjusted Z-scores, and a positive correlation was found between $A L$ and CCR. The decreasing power of the cornea appears to play an emmetropizing role in response to increasing $A L$; however, this compensatory capacity reportedly reaches its limit at an $A L / C C R$ ratio of $3^{32}$. In the linear regression model used in the current study, the AL/CCR ratio exceeded 3 when the Z-AL score was above 2.16. Though, the correlation between AL and preoperative spherical equivalent was not studied because the displaced and deformed lens can affect the total refractive power.

Nevertheless, we hypothesized that in the postoperative eyes with centered and stabilized IOL positions, a long-term myopic shift is expected in the developing eyes when the Z-AL exceeds 2.16. Therefore, the selection of IOL power deserves more careful consideration in those patients in order to achieve the refractive target. Microspherophakia and megalocornea are sometimes found in eyes of MFS patients 33,34. To the best of our knowledge, the prevalence of these two comorbidities in MFS patients with bilateral EL have never been reported. Our study indicated that about $10 \%$ of eyes of MFS patients were 
complicated by microspherophakia, the AL of which seemed to be greater than that of normal eyes but not to a statistically significant degree. Megalocornea was found in about 7\% of MFS eyes. Although WTW measurements did not change proportionally with AL growth, MFS eyes complicated by megalocornea had significantly larger $\mathrm{AL}$ on average. Our data show there was high corneal astigmatism in the eyes of MFS patients, which was consistent with previous studies ${ }^{35,36}$. Additionally, we found that patients with smaller AL generally had higher corneal astigmatism. Thus, residual astigmatism from the cornea after lens aspiration and IOL implantation, especially in MFS eyes with smaller AL, is a challenge for postoperative visual rehabilitation.

The results of this study must be assessed within the context of its limitations. First, the data are not a complete representation of MFS patients and only relate to surgical patients with bilateral EL, which prevented us from detecting differences in eyes with unilateral EL or without EL. Second, the external controls used in our study were school-based, cross-sectional studies of young Chinese individuals from a large sample size. The conclusions should be viewed with caution for other ethnic populations. Third, adult patients were excluded from the current study, as AL were shown to be stable in adults ( $>30$ years old) with MFS during a 10-year follow up ${ }^{37}$. The cross-sectional nature of our study also limited our ability to track changes in $A L$ data over time. Despite these limitations, the results of this study describe the overall distribution of AL in the eyes of Chinese MFS patients under 20 years old with bilateral EL.

In conclusion, the mean AL of MFS was found to be longer than the age-matched controls, especially in the eyes complicated by megalocornea. But one-third of patients still had an AL below the normative average. The considerable individual variation indicates that young patients with bilateral EL but small AL should not be excluded from MFS diagnoses without systematic examinations. The Z-scores normalized the age difference in AL and other parameters by comparing age-matched published normative values, and this technique facilitates the further study of the influence factors of AL in young patients of different ages. A full understanding of the AL will be helpful for surgical planning and complication monitoring.

\section{Methods}

\section{Patient Eligibility}

The study was approved by the Human Research Ethics Committee of the Eye \& ENT Hospital of Fudan University and carried out in accordance with the Declaration of Helsinki. Informed consent was obtained from all patients and from the patients' guardians for those under 18 years old. The inclusion criteria were as follows: (1) patients diagnosed as having MFS or possible MFS according to the Ghent 2 nosology 14; (2) patients under 20 years old (because they are defined as 'young individuals' in Ghent 2 nosology, who have different diagnostic criteria); (3) patients with bilateral EL under slit-lamp examination during complete pupillary dilation. Patients with the following features were excluded from the study: (1) lens dislocated into the anterior chamber or vitreous body; (2) a history of traumatic lens dislocation; (3) pre-existing ocular comorbidities, such as congenital cataract, retinal tears, retinal 
detachment, corneal ectasia, or glaucoma; (4) the presence of other connective tissue diseases. Both eyes were selected because we found a significant difference in the AL between the two eyes of each participant by paired t-test $(P=0.014)$. The recorded patients' ages were the ages at examination.

\section{Ophthalmic Examinations}

The participants were examined by board-certified ophthalmologists, and their medical and family histories were evaluated. All patients were examined for EL by slit-lamp examination under complete pupillary dilation. Bilateral EL was defined as the displacement of the lens from its normal position in both eyes. The AL, CCR, WTW, and corneal astigmatism were measured using partial coherence interferometry (IOLMaster 500, Carl Zeiss Meditec AG, Jena, Germany). Five valid AL readings and three readings of both CCR and WTW were taken.

\section{Z-Score Calculation}

The AL and other ocular biometrics were compared to age-matched normative data published in the literature that were cross-sectional, epidemiological investigations of the Chinese population. The Z-score of $A L$ (Z-AL) was calculated using the formula below: $Z-A L=$ (measured $A L$ - normative $A L$ )/ normative standard deviation (SD). The Z-score describes the position of a raw value in terms of its distance from the mean when measured in standard deviation units. For example, a Z-score $\geq 2.0$ corresponds to a measurement $\geq 2.0$ SD above normal. For patients of 4-18 years of age, the AL, CCR, and WTW were compared with normative measurement data in the Shandong Children Eye Study ${ }^{15-17}$. For those over 18 years old, the measurements for 18-year-old children in the same study were employed as reference data. For 3-year-old patients, the Shanghai Pudong Eye Study was used as the reference 18 (Supplementary Table S1 online). Because of a lack of normative measurements for 2-year-olds in the Chinese population, the Z-score was not analyzed in one patient (two eyes). The Z-scores for other biometric parameters, such as Z-CCR and Z-WTW, were calculated in a similar manner.

\section{Statistical Analysis}

Continuous variables were described in mean $\pm S D$, and categorical variables were described in counts or proportions as appropriate. The distributions for age and ocular biometric parameters were tested for normality with the Kolmogorov-Smirnov test. The one-way analysis of variance or Kruskal-Wallis test was used, as appropriate, to compare the AL among different age groups. For independent samples, the ttest or Wilcoxon test was used to analyze differences in the AL among patients with different factors, including gender, family history, and comorbidities. And the paired t-test or paired Wilcoxon test was used to evaluate the difference in AL between the left eye and the right eye. A logarithmic correlation between $\mathrm{AL}$ and age was calculated for MFS patients. Correlations between the Z-score of AL and Z-scores of other biometric variables were analyzed using Spearman's correlation. All analyses were 2-tailed, and the statistical significance was set as a $P$-value of less than 0.05 . Statistical analyses were performed using SPSS version 22.0 (IBM Corp., Armonk, NY, USA).

\section{Declarations}




\section{Acknowledgement}

This study was supported by the National Natural Science Foundation of China (grant no. 81770908), the National Natural Science Foundation of China (grant no. 82070943), the Shanghai Science and Technology Commission (Scientific Innovation Action Plan, grant no.18411965200), and the Shanghai Science and Technology Commission (Scientific Innovation Action Plan, grant no. 20Y11911000). The sponsors played no role in the study design, data collection, data analysis, manuscript preparation, nor the decision to submit the manuscript for publication.

\section{Author contribution}

Z.X.C. and J.H.C. were involved in study design, data collection and manuscript preparation. M.Z. and J.L.Z. participated in data acquisition. M.D. and T.H.C critically reviewed the manuscript. Y.H.J. and Y.X.J. supervised the project. All the authors have read and approved the final manuscript.

\section{Data availability statement}

The datasets generated and/or analyzed during the present study are not publicly available (obtained from Eye \& ENT Hospital, of Fudan University, Shanghai repository), but are available from the corresponding author upon reasonable request.

\section{Competing financial interests}

The authors declare no competing financial interests

\section{References}

1. Nemet, A.Y., Assia, E.I., Apple, D.J. \& Barequet, I.S. Current concepts of ocular manifestations in Marfan syndrome. Surv Ophthalmo/ 51, 561-75 (2006).

2. Groth, K.A. et al. Prevalence, incidence, and age at diagnosis in Marfan Syndrome. Orphanet $J$ Rare Dis 10, 153 (2015).

3. Fuchs, J. Marfan syndrome and other systemic disorders with congenital ectopia lentis. A Danish national survey. Acta Paediatr 86, 947-52 (1997).

4. De Paepe, A., Devereux, R.B., Dietz, H.C., Hennekam, R.C. \& Pyeritz, R.E. Revised diagnostic criteria for the Marfan syndrome. Am J Med Genet 62, 417-26 (1996).

5. Kainulainen, K., Pulkkinen, L., Savolainen, A., Kaitila, I. \& Peltonen, L. Location on chromosome 15 of the gene defect causing Marfan syndrome. N Engl J Med 323, 935-9 (1990). 
6. De Maria, A., Wilmarth, P.A., David, L.L. \& Bassnett, S. Proteomic Analysis of the Bovine and Human Ciliary Zonule. Invest Ophthalmol Vis Sci 58, 573-585 (2017).

7. Jones, W., Rodriguez, J. \& Bassnett, S. Targeted deletion of fibrillin-1 in the mouse eye results in ectopia lentis and other ocular phenotypes associated with Marfan syndrome. Dis Model Mech 12(2019).

8. Maumenee, I.H. The eye in the Marfan syndrome. Trans Am Ophthalmol Soc 79, 684-733 (1981).

9. Salchow, D.J. \& Gehle, P. Ocular manifestations of Marfan syndrome in children and adolescents. European journal of ophthalmology 29, 38 (2019).

10. Gehle, P. et al. Biometric and structural ocular manifestations of Marfan syndrome. PloS one 12, e0183370 (2017).

11. Drolsum, L., Rand-Hendriksen, S., Paus, B., Geiran, O.R. \& Semb, S.O. Ocular findings in 87 adults with Ghent-1 verified Marfan syndrome. Acta Ophthalmologica 93, 46-53 (2015).

12. Konradsen, T.R., Koivula, A., Kugelberg, M. \& Zetterström, C. Corneal curvature, pachymetry, and endothelial cell density in Marfan syndrome. Acta Ophthalmologica 90, 375-379 (2012).

13. Heur, M. et al. The Value of Keratometry and Central Corneal Thickness Measurements in the Clinical Diagnosis of Marfan Syndrome. American Journal of Ophthalmology 145, 997-1001.e1 (2008).

14. Loeys, B.L. et al. The revised Ghent nosology for the Marfan syndrome. Journal of Medical Genetics 47, 476-485 (2010).

15. Zhang, Y.Y. et al. Corneal Curvature Radius and Associated Factors in Chinese Children: The Shandong Children Eye Study. PLOS ONE 10, e0117481 (2015).

16. Lu, T.L. et al. Axial Length and Associated Factors in Children: The Shandong Children Eye Study. Ophthalmologica 235, 78-86 (2016).

17. Jiang, W.J. et al. Corneal diameter and associated parameters in Chinese children: the Shandong Children Eye Study. Clinical \& Experimental Ophthalmology 45, 112-119 (2017).

18. Zhao, K. et al. Axial length/corneal radius of curvature ratio and refractive development evaluation in 3- to 4-year-old children: the Shanghai Pudong Eye Study. International journal of ophthalmology 12 , $1021-1026$ (2019).

19. Wilson, M.E. \& Trivedi, R.H. Axial length measurement techniques in pediatric eyes with cataract. Saudi J Ophthalmol 26, 13-7 (2012).

20. Zhang, Y. et al. Distribution of axial length in Chinese congenital ectopia lentis patients: a retrospective study. BMC Ophthalmol 17, 113 (2017).

21. Tideman, J. et al. Axial length growth and the risk of developing myopia in European children. Acta Ophthalmol 96, 301-309 (2018).

22. Lin, H. et al. Distribution of Axial Length before Cataract Surgery in Chinese Pediatric Patients. Scientific reports 6, 23862 (2016).

23. Faivre, L. et al. Clinical and Molecular Study of 320 Children With Marfan Syndrome and Related Type I Fibrillinopathies in a Series of 1009 Probands With Pathogenic FBN1 Mutations. Pediatrics 
(Evanston) 123, 391-398 (2009).

24. Roman, M.J., Devereux, R.B., Kramer-Fox, R. \& O'Loughlin, J. Two-dimensional echocardiographic aortic root dimensions in normal children and adults. Am J Cardio/ 64, 507-12 (1989).

25. Devereux, R.B. et al. Normal limits in relation to age, body size and gender of two-dimensional echocardiographic aortic root dimensions in persons $\geq 15$ years of age. Am J Cardio/ 110, 1189-94 (2012).

26. Fan, F., Luo, Y., Liu, X., Lu, Y. \& Zheng, T. Risk factors for postoperative complications in lensectomyvitrectomy with or without intraocular lens placement in ectopia lentis associated with Marfan syndrome. British Journal of Ophthalmology 98, 1338-1342 (2014).

27. Xu, W. et al. Comparative data on SD-OCT for the retinal nerve fiber layer and retinal macular thickness in a large cohort with Marfan syndrome. Ophthalmic genetics 38, 34-38 (2017).

28. Chandra, A. et al. The revised ghent nosology; reclassifying isolated ectopia lentis. Clin Genet $\mathbf{8 7}$, 284-7 (2015).

29. Sakai, L.Y. \& Keene, D.R. Fibrillin protein pleiotropy: Acromelic dysplasias. Matrix Bio/ 80, 6-13 (2019).

30. Faivre, L. et al. Effect of mutation type and location on clinical outcome in 1,013 probands with Marfan syndrome or related phenotypes and FBN1 mutations: an international study. Am J Hum Genet 81, 454-66 (2007).

31. Chen, J. et al. Age Differences in Axial Length, Corneal Curvature, and Corneal Astigmatism in Marfan Syndrome with Ectopia Lentis. Journal of Ophthalmology 2018, 1-8 (2018).

32. GONZÁLEZ BLANCO, F., SANZ FERŃANDEZ, J.C. \& MUŃOZ SANZ, M.A. Axial Length, Corneal Radius, and Age of Myopia Onset. Optometry and vision science 85, 89-96 (2008).

33. Farat, J.G., Queiroz, M. \& Lottelli, A.C. Bilateral clear lens extraction and intraocular lens implantation in a child with microspherophakia and Marfan syndrome. Arq Bras Oftalmol 82, 62-64 (2019).

34. Consul, B.N., Mehrotra, A.S. \& Mathur, G.B. Marfan's syndrome with bilateral megalocornea and subluxated cataractous lenses. J All India Ophthalmol Soc 14, 262-3 (1966).

35. Zhang, Y. et al. Analysis of Corneal Astigmatism before Surgery in Chinese Congenital Ectopia Lentis Patients. Curr Eye Res 43, 972-976 (2018).

36. Chen, J. et al. Corneal Curvature, Astigmatism, and Aberrations in Marfan Syndrome with Lens Subluxation: Evaluation by Pentacam HR System. Sci Rep 8, 4079 (2018).

37. Sandvik, G.F. et al. Ten-year reinvestigation of ocular manifestations in Marfan syndrome. Clin Exp Ophthalmol 47, 212-218 (2019).

\section{Figures}




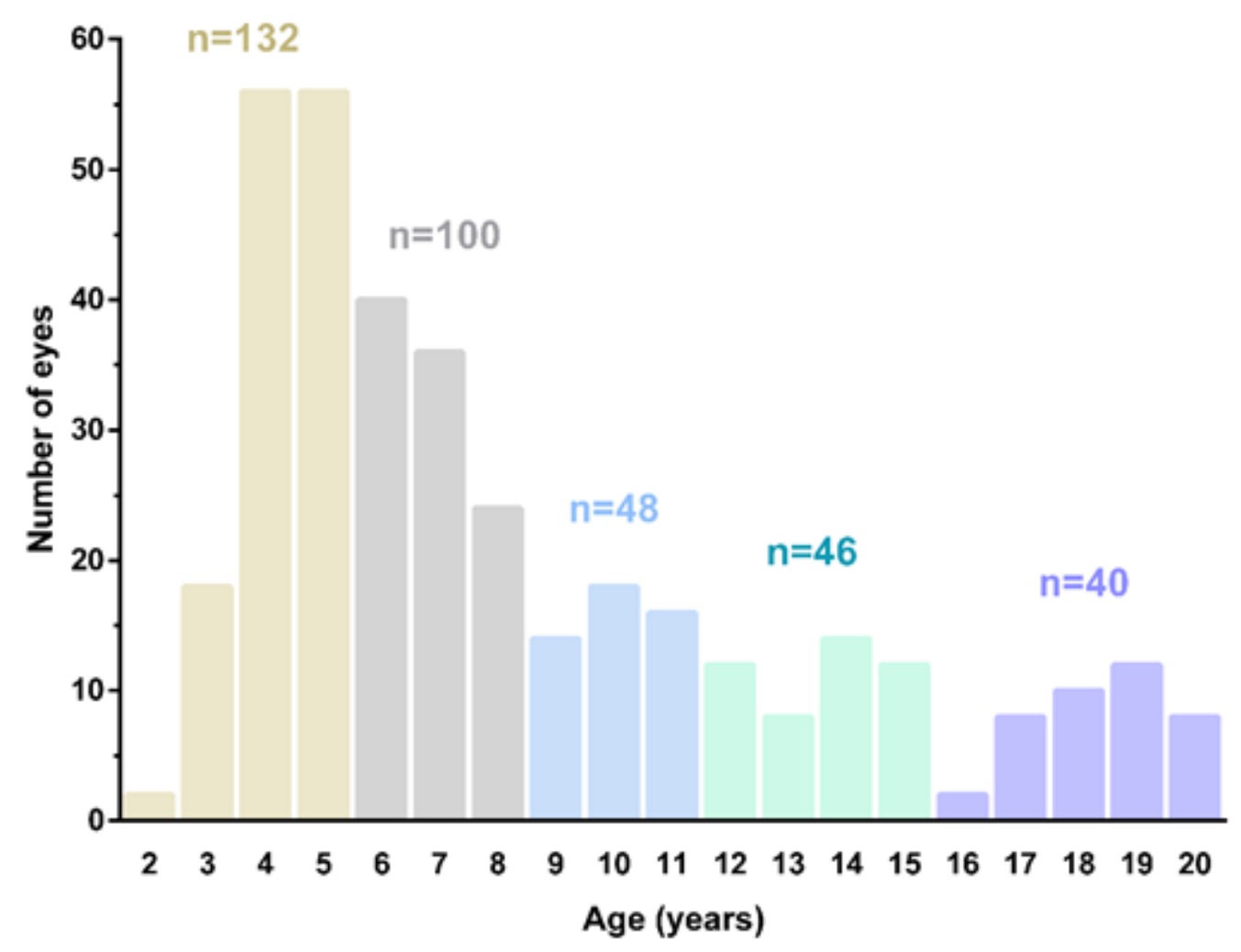

Figure 1

The age distribution of MFS patients with bilateral EL Subdivided groups were marked by different colors: $<5$ years old (yellow), 6-8 years old (gray), 9-11 years old (blue), 12-15 years old (cyan), and 16-20 years old (purple). The number of eyes ( $n$ ) in each group was labeled. EL, ectopia lentis. MFS, Marfan syndrome.

a

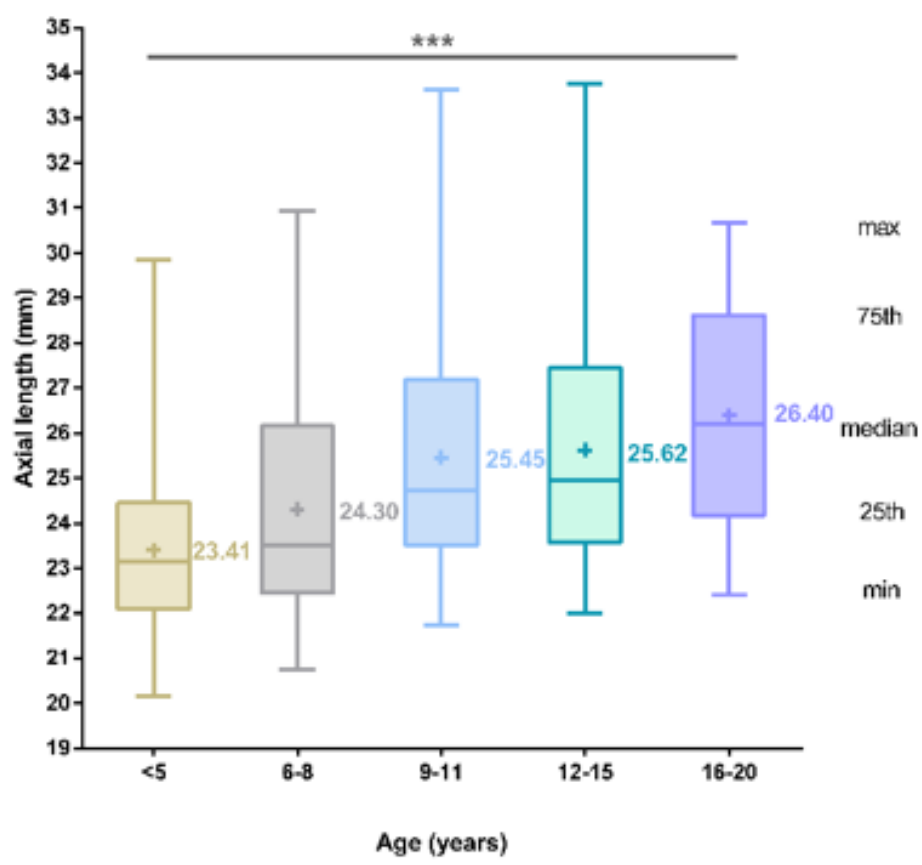

b

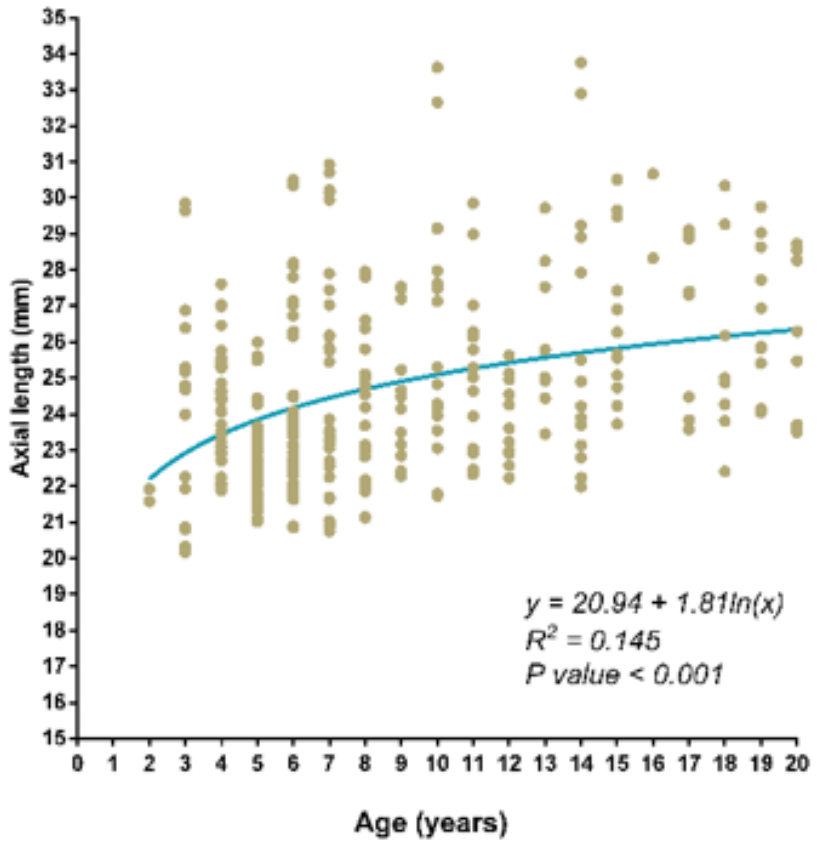


Figure 2

Differences in AL among MFS patients with bilateral EL of various ages (a) Boxplots showing the distribution and median of $A L$ in different age subgroups. The mean value is denoted by ' + '. ${ }^{* \star *} \mathrm{P}<0.001$ (Kruskal-Wallis test). (b) Scatterplot of AL by patients' ages and fitted logarithmic curve. AL, axial length. EL, ectopia lentis. MFS, Marfan syndrome.

a

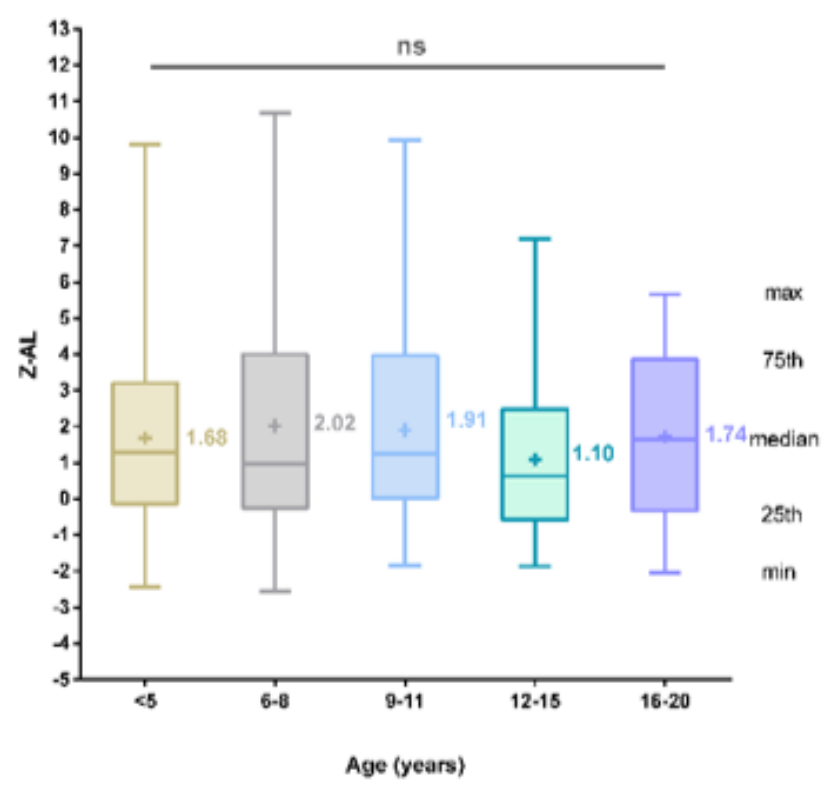

b

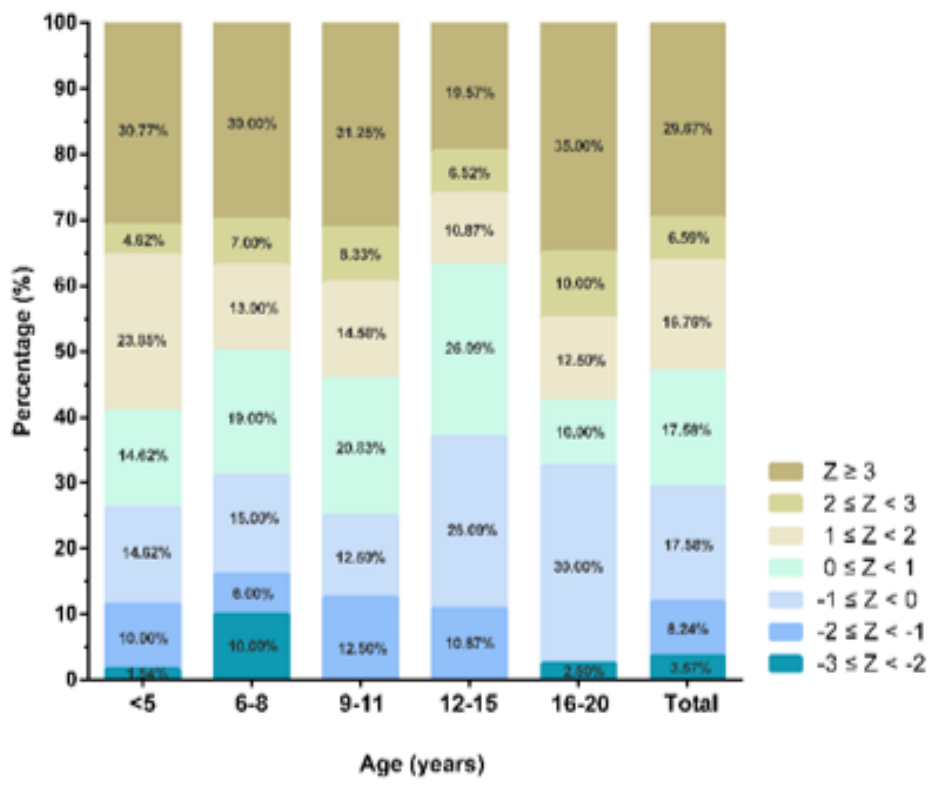

\section{Figure 3}

Differences in the Z-AL score of MFS patients with bilateral EL of different ages (a) Boxplots showing the distribution and median of Z-AL scores in different age subgroups. ns, $P>0.05$ (Kruskal-Wallis test). (b) Proportions of Z-AL scores in different age groups. AL, axial length. EL, ectopia lentis. MFS, Marfan syndrome. 
a

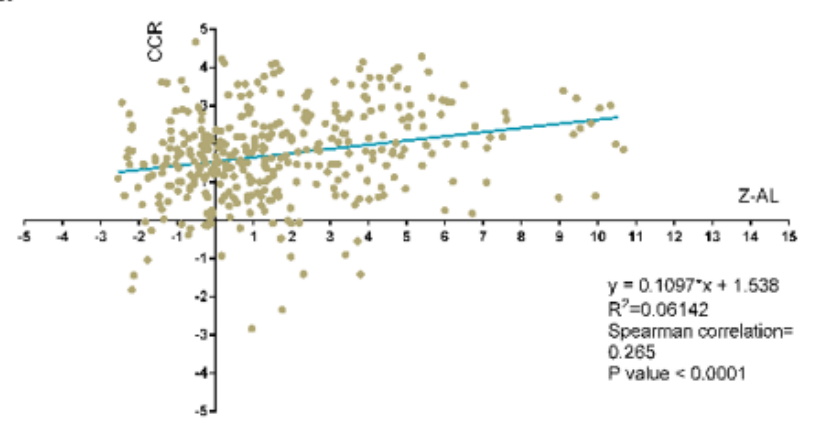

C

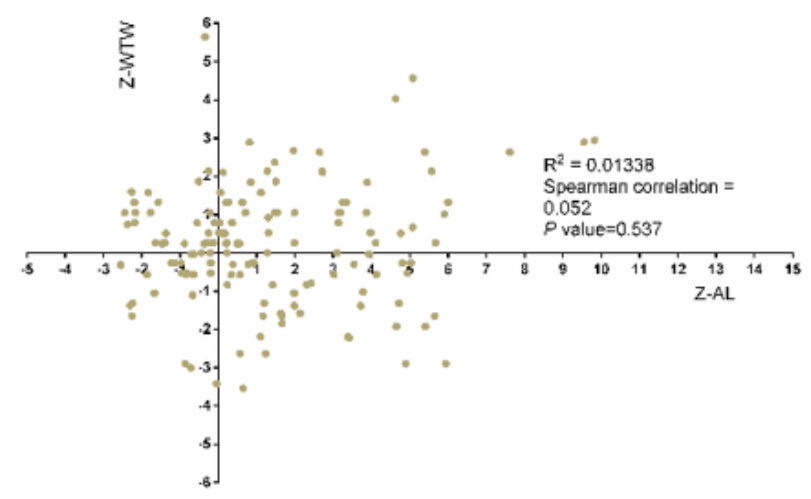

b

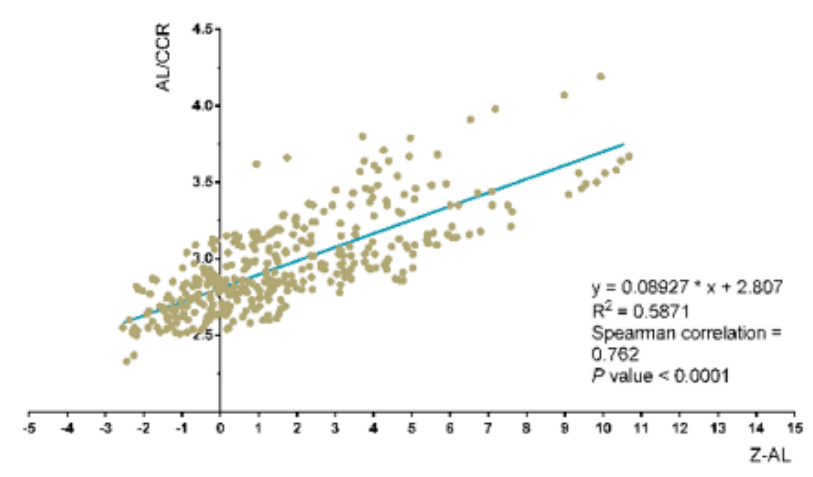

d

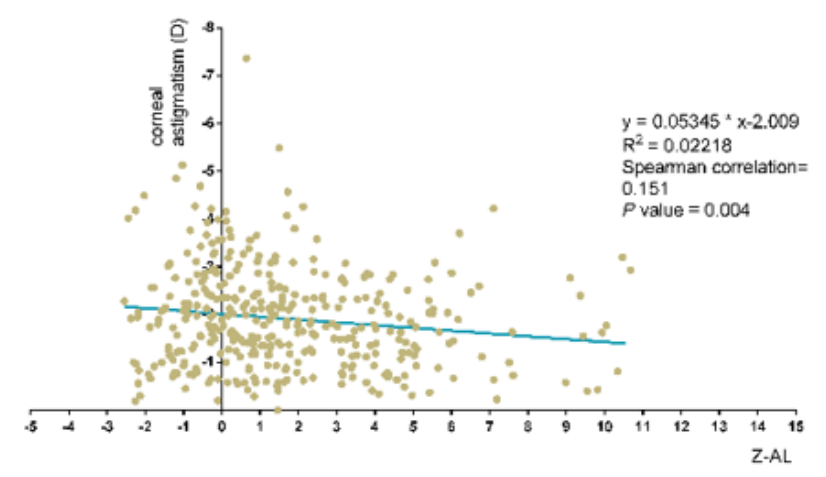

\section{Figure 4}

Scatterplots showing correlations (Spearman's correlation) between the Z-AL score and Z-CCR score (a), AL/CCR ratio (b), Z-WTW score (c), and corneal astigmatism (d) in MFS patients with bilateral EL $A L$, axial length. CCR, corneal curvature radius. MFS, Marfan syndrome. WTW, white-to-white horizontal corneal diameter.

\section{Supplementary Files}

This is a list of supplementary files associated with this preprint. Click to download.

- AnalysisofALinMFSeyeswithBELbyZscoreSupplementary.pdf 